\title{
MOLECULAR CHARACTERIZATION OF Begomovirus INFECTING YARD LONG BEAN (Vigna unguiculata subsp. sesquipedalis L.) IN JAVA, INDONESIA
}

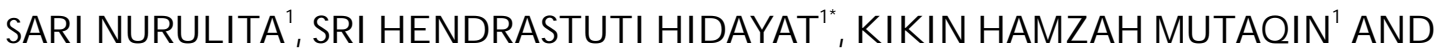 \\ JOHN THOMAS
}

\author{
${ }^{1}$ Departmett of Plant Protetion, Faalty of Agiailture, Institut Petanian Bogr, Bogor 16680, Indonesia \\ ${ }^{2}$ UQueendandAlltrancefor AgiaitureandFoodImovation(QAAFI) \\ University of Queendand, St. Luia Campus Bisbane, Australia
}

Received 21 May 2014/ Accepted 6 June 2015

\begin{abstract}
Begmoinuswas identified as one of the causal agents associated with yellow mosaic disease on yard long bean (Vigna ungiiaulata subsp. sequipedalis L.) in Java. Previous study reported that Beegmoinus has infected several Leguminosae in South Asia. Several Begmoinuseshave been reported to infect important crops in Indonesia. Those Begmovinuses were characterized based on nucleotide sequences. This study was conducted to identify and characterize Beemowinustaken from yard long bean samples in Java based on specific genome property of common region. Three main activities were conducted: i) sample collection in yard long bean fields located in Central Java (Tegal, Magelang, and Klaten), Yogyakarta (Sleman) and West Java (Bogor and Subang) provinces; ii) virus detection using I-ELISA, PCR and sequencing; iii) molecular characterization of Begmoinususing software BioEdit v.7.0.5 and MEG A 6.06. Yellow mosaic disease was found in almost all fields. Infection of Pdyinsand Beegnoinuswas detected using I-ELISA and PCR, respectively. Both viruses were detected as either single or mixed infection. Samples collected from Tegal, Klaten, Magelang, Subang and Bogor were positively infected by Beegmoinusbased on specific viral DNA amplification. Sequence analysis indicated that Begmoinusinfecting yard long bean is Mungbean Yellow Mosaic India Virus (MY MIV) and it belongs to the same group with MYMIV from Bangladesh, India, Pakistan and Nepal. Further analysis showed the conserved region of Begmoinus around Common Region, i.e. "TATA box" sequence, hair pin loop structure, repetitive sequence and the conserved nonanucleotide sequence TAATATTAC were also determined. This is the firstreport of MY MIV infection in Indonesia.
\end{abstract}

Keywords: Begomoins, common region, DNA sequencing, I-ELISA, MYMIV, PCR, yard long bean

\section{INTRODUCTION}

A yellow mosaic disease outbreak was reported in yard long bean (Vigna ungiaulata subsp. sesquipedalis L.) growing area in Java since 2008 (Damayanti $\notin$ al. 2009). The disease was associated with infection of Bean Common Mosaic Virus (BCMV), Cucumber Mosaic Virus (CMV) and Begmouinus (D amayanti \& al. 2009; Hidayat 2011, personal communication; Tsai eal. 2013). At the same time, similaryellow mosaic disease caused by Beegmoinuswas first reported from Pakistan (Ilyas eal. 2010) and Nepal (Shahid tal. 2012).

Beegmoinus is a member of Geminivinus group which is transmitted in nature by whitefly Bemisia tabad G en. (Hemiptera: Aleyrodidae). Molecular

\footnotetext{
*Corresponding author : srihendrastutihidayat@ gmail.com
}

characteristics of its genome is very unique, having one or two circular single stranded D NA (2.6-2.7 kb) and represented as monopartite or bipartite, respectively (Hull 2002). Serious crop diseases caused by infection of Bexmovinus have been reported in Indonesia. Pepper Yellow Leaf Curl Virus (PepYLCV), Tomato Yellow Leaf Curl Virus (TYLCV), and Tobacco Leaf Curl Virus (TLCV) are considered as the major factor causing yield loss on chilli pepper, tomato and tobacco, respectively (Aidawati \&al. 2005; Hidayat et al. 2006; 2008). D amage and potential yield loss in yard long bean caused by Beemoinusshould be anticipated by establishing detection method and disease control strategy. To do so, basic information regarding molecular and biological characters of the virus is required. 
Method for detection of Begomovinus is commonly based on polymerase chain reaction (PCR) technique using universal primers. D egenarate primers PAL1v1978/ PAR1c715 was first used by Rojas \&al. (1993) as universal primer to detect several Geminivinusesfrom infected plants belong to Solanaceae, Leguminosae, Euphorbiaceae and Malvaceae from South A merica. Since then, the primer pair has been used to identify and characterize various members of Begmovinus Fragment of Beegmoinus D NA amplified by this pair of primers covers part of AL1 region (replicase gene), common region and intergenic region and part of $A R 1$ region (coat protein gene). Molecular characters of Begmoinus can be determined by analyzing this DNA fragment sequences due to its conserved and diversed region. Common region for each Begmoinus is different, except loop region on hairpin structure, 5'-TAATATTAC-3' which is known as the conserved nonanucleotide region (Lazarowitz 1987). Beegmoinus possessing $>92 \%$ similarity on their hairpin loop structure can be grouped as one species (Fauquet $\notin a l .2005$ ).

This paper explained the attempts to identify and characterize Begmovinusfrom yard long bean samples in Java based on specific genome property of common region.

\section{MATERIALS AN D METHODS}

\section{Collection of Field Samples}

Samples collection was conducted in West Java (Bogor and Subang), Central Java (Tegal, Klaten and Magelang) and Yogyakarta (Sleman). Leaf samples were collected from the plants age 6-9 WAP using purposive sampling method based on yellow mosaic symptom described by D amayanti eal. (2009) and Ilyas \&al. (2010). Fresh tissue was directly subjected for virus detection and the remaining were stored as isolate collection in the laboratoryat $-80^{\circ} \mathrm{C}$.

\section{Virus Detection by Indirect Enzyme-linked ImmunosorbentAssay (I-ELISA)}

Two major viruses on yard long bean i.e. Poyinus and CMV were detected from leaf samples using I-ELISA commercial kit following manufacturer protocol (Leibniz-Institut DSMZ $\mathrm{G} \mathrm{mbH}$, G ermany). Infected leaves was ground in coating buffer (1/ 10, v/v). Coating buffer (pH 9.6) containing $15 \mathrm{mM} \mathrm{Na}_{2} \mathrm{CO}_{3}(1.59 \mathrm{~g}), 2.38 \mathrm{mM}$ $\mathrm{NaHCO}_{3}(2.93 \mathrm{~g}), 3.08 \mathrm{mM} \mathrm{NaN}_{3}(0.20 \mathrm{~g})$ and $\mathrm{H}_{2} \mathrm{O}$ was added to final volume of $1 \mathrm{~L}$. Aliquots of each sample $(100 \mu \mathrm{L})$ was dispensed to each microtitters' well, then incubated overnight at $4{ }^{\circ} \mathrm{C}$. Microtitter plate was washed 8 times using PBS-Tween $(0.137 \mathrm{M} \mathrm{NaCl}(8.0 \mathrm{~g}), 1.47 \mathrm{mM}$ $\mathrm{KH}_{2} \mathrm{PO}_{4}(0.2 \mathrm{~g}), 8.1 \mathrm{mM} \mathrm{Na}_{2} \mathrm{HPO}_{4}(1.15 \mathrm{~g}), 2.68$ $\mathrm{mM} \mathrm{KCl}(0.2 \mathrm{~g}), 3.08 \mathrm{mM} \mathrm{NaN}_{3}(0.2 \mathrm{~g})$, add $\mathrm{H}_{2} \mathrm{O}$ up to $1 \mathrm{~L} \mathrm{pH} 7.4$ and add $0.5 \mathrm{ml}$ Tween $20 / \mathrm{L}$ ). Plates were dried up by tapping upside down on tissue paper. Blocking solution (2\% skim milk diluted in PBS-Tween) was added $100 \mu \mathrm{L}$ to each well then incubated at $37{ }^{\circ} \mathrm{C}$ for 30 minutes. Blocking solution was removed and plate was tapped dry. First antibody (IgG) was diluted in conjugate buffer (PBS-Tween containing 2\% PVP (Serva PVP-15 polyvinyl pyrrolidon) and $0.2 \%$ egg albumin) according to manufacturer recommendation, then $100 \mu \mathrm{L}$ conjugate buffer contains IgG was added to each well, incubated at $37{ }^{\circ} \mathrm{C}$ for $2-4$ hours, then washed by PBS-Tween. Second antibody (IgG-AP) was diluted in conjugate buffer according to manufacturer recommendation, then $100 \mu \mathrm{L}$ conjugate buffer containing IgG-AP was added to each well, incubated at $37^{\circ} \mathrm{C}$ for 2 hours then washed using PBS-Tween. Substrate (10 mg p-nitrophenyl phosphate) was dissolved in substrate buffer (97 $\mathrm{mL}$ diethanolamine, $600 \mathrm{~mL} \mathrm{H}_{2} \mathrm{O}, 3.08 \mathrm{mM} \mathrm{NaN}_{3}$ $(0.2 \mathrm{~g})$, adjust to $\mathrm{pH} 9.8$ with $\mathrm{HCl}$ and make up to $1 \mathrm{~L}$ with $\mathrm{H}_{2} \mathrm{O}$ ) added $100 \mu \mathrm{L}$ to each well. Plate was incubated in the room temperature under low light intensity and reaction was evaluated. Positive reaction was qualitatively indicated by appearance of yellow color and quantitatively determined by measuring absorbance value at $405 \mathrm{~nm}$ wave length, i.e. two times absorbance value of negative control (healthy plant), using ELISA reader (Biorad 550).

\section{Virus Detection by PCR}

Total viral DNA was isolated from infected leaf following a procedure described by Doyle and D oyle (1987) with minor modification. Fresh tissue $(0.1 \mathrm{~g})$ was ground with liquid nitrogen to powder, $500 \mu \mathrm{L}$ of CTAB buffer $(10 \%$ Cetyltrimethyl-ammonium bromide $(100 \mathrm{~mL}), 0.1 \mathrm{M}$ Tris-HCl pH 8 (100 mL), 0.05 M EDTA (50 mL), $0.5 \mathrm{M} \mathrm{NaCl}(126 \mathrm{~mL}), 1 \% \beta$-mercapto-ethanol 
(10 $\mathrm{mL}$ and added $\mathrm{H}_{2} \mathrm{O}$ up to $1 \mathrm{~L}$ ) was added and the sap was transferred to $1.5 \mathrm{~mL}$ clean tube. The sap wasincubated in water bath at $65^{\circ} \mathrm{C}$ for 1 hour, shaked every 10 minutes to separate lipid and protein. Five hundred microliter of chloroform/ iso-amyl alcohol (24:1, v/v) was added to the liquid, then the tube was vortexed for 5 minutes and centrifuged at 14,000 rpm for 15 minutes. The supernatant was pipetted to $1.5 \mathrm{~mL}$ clean tube, $1 / 10$ volume of $3 \mathrm{M}$ ammonium acetate and 2/3 volume of isopropanol was added, respectively. The liquid was mixed gently then incubated overnight at $-20^{\circ} \mathrm{C}$ or 4 hours at room temperature. After incubation, the liquid was centrifuged at $12,000 \mathrm{rpm}$ for 10 minutes to precipitate DNA and then was discarded flowthrough. The pellets were washed with $500 \mu \mathrm{L}$ of $70 \%$ ethanol, centrifuged at 8,000 rpm for 5 minutes and dried in room temperature after discarding the flow through. The dried pellets containing total DNA were dissolved in 50 to 100 $\mu \mathrm{L}$ of nuclease free water or TE buffer, $\mathrm{pH} 8$ and the D NA was ready for PCR.

Amplification of viral DNA was conducted following method described by Rojas \&al. (1993) to confirm geminivirus infection. PCR reaction contained 10xPCR Buffer, $25 \mathrm{mM} \mathrm{MgCl}_{2}, 2.5 \mathrm{mM}$ dNTPS, 10 $\mu \mathrm{M}$ each of primer PAL1v1978 and PAR1c715, Taq polymerase $(5 \mathrm{U} / \mu \mathrm{L}), 1 \mu \mathrm{L}$ of DNA and the reaction was adjusted to $25 \mu \mathrm{L}$ with nuclease free water. Amplifications was performed in GeneAmp PCR System 9700 machine with 5 minutes at $94^{\circ} \mathrm{C}$ for pre-heating, followed by 30 cycles of denaturation (1 minute at $\left.94^{\circ} \mathrm{C}\right)$, annealing $\left(1\right.$ minute at $\left.50^{\circ} \mathrm{C}\right)$ and extension ( 3 minutes at $72^{\circ} \mathrm{C}$ ). The last cycle was followed by $72{ }^{\circ} \mathrm{C}$ for 3 minutes and decreased at $4^{\circ} \mathrm{C}$. Agarose gel electrophoresis was used to visualize PCR products.

\section{DNA Sequencing}

Viral DNA fragments obtained from direct PCR amplification were sent to PT Genetika Science, Indonesia and Australian Genome Research Facility, Australia, respectively for D NA sequencing. Sequence data were compared with other sequences from G enBank (NCBI 2013) and analysed using software programs BioEdit V.7.0.5, CLC Sequence Viewer 7, and MEGA 6.06 .

\section{RESULTS AND DISCUSSION}

\section{Identification of Begomovirus from Field Samples}

Incidence of yellow mosaic disease was very high i.e. $80 \%$ to $100 \%$ in most growing areas. Infected plants were easily recognized in the field based on visual symptoms. Three main symptoms of yellow mosaic disease in the field were observed i.e. 1) yellowing; 2) yellowing with green spot; and 3) mosaic vein banding (Fig. 1 and Table 1). The most common symptom found in every field was yellowing. Yellowing with green spot was thought as early symptom before it developed into yellowing. Further severe infection caused smaller pods and leaves. These types of symptom had also been reported as typical symptoms of yellow mosaic disease of yard long bean in South Asia (Ilyas \& al. 2010). Bexgmoinus was detected in 11 infected plant samples (Fig. 2) showing yellowing and yellowing with green spot symptoms from all locations. However, Begomovirus fragment was not successfully amplified from samples showing mosaic vein banding from Subang Viral detection using I-ELISA revealed the infection of Potyinus
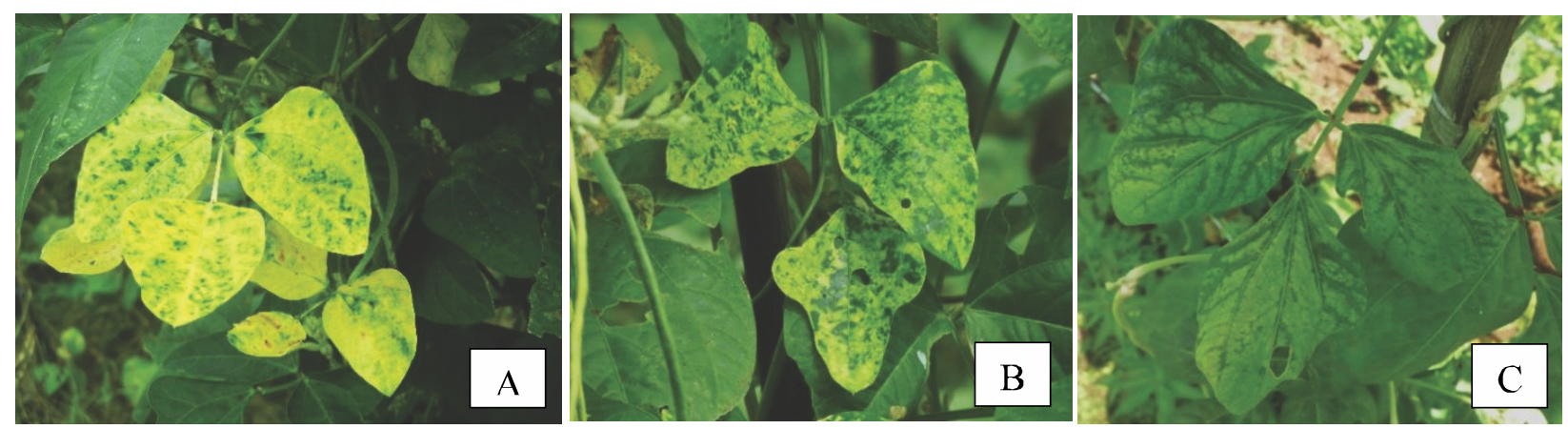

Figure 1. Symptoms of yellow mosaic disease on yard long bean: (A) yellowing; (B) yellowing with green spot; (C) mosaic vein banding 
Table1. D etection of CucumberMosaic Virus, Potyinusand Beegmoinusfrom leaf samples collected in wet season 2012 from various locations using I-ELISA and PCR ${ }^{\mathrm{a}}$

\begin{tabular}{|c|c|c|c|c|c|}
\hline \multirow{2}{*}{ Location } & \multirow{2}{*}{ Code of Isolates } & \multirow{2}{*}{ Symptoms description } & \multicolumn{2}{|c|}{ I-ELISA ${ }^{b}$} & \multirow{2}{*}{$\begin{array}{c}\text { PCR } \\
\text { Begmainus }\end{array}$} \\
\hline & & & $\mathrm{CMV}$ & Pdyinus & \\
\hline \multirow[t]{2}{*}{ Tegal } & Tegal 1 & Yellowing & - & + & + \\
\hline & Tegal 2 & Yellowing mosaic & - & - & + \\
\hline \multirow[t]{2}{*}{ Klaten } & Klaten 1 & Yellowing & - & - & + \\
\hline & Klaten 2 & Yellowing mosaic & - & - & - \\
\hline \multirow[t]{2}{*}{ Sleman } & Sleman 1 & Yellowing & - & + & + \\
\hline & Sleman2 & Yellowing mosaic & - & + & + \\
\hline \multirow[t]{3}{*}{ Magelang } & Magelang 1 & Yellowing mosaic & - & - & + \\
\hline & Magelang 2 & Yellowing & - & + & + \\
\hline & Magelang 3 & Yellowing mosaic & - & - & + \\
\hline \multirow[t]{4}{*}{ Subang } & Subang 1 & Mosaic vein banding & - & + & - \\
\hline & Subang 2 & Mosaic vein banding & - & + & - \\
\hline & Subang 3 & Mosaic vein banding & - & - & - \\
\hline & Subang 4 & Yellowing & - & - & + \\
\hline \multirow[t]{2}{*}{ Bogor } & Bogor 1 & Yellowing & - & - & + \\
\hline & Bogor 2 & Yellowing & - & - & + \\
\hline
\end{tabular}

${ }^{\mathrm{a}}$ Most plants were in generative stage when samples was collected

b - = not detected; + = detected

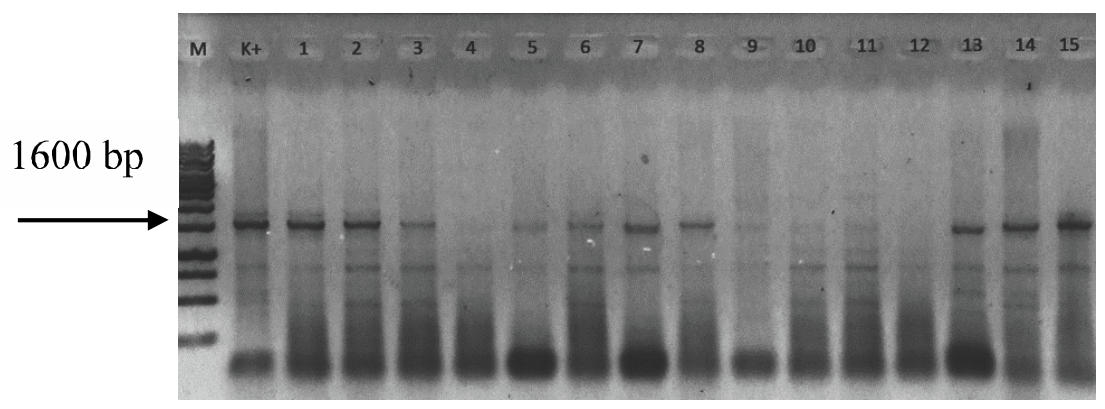

Figure 2. Visualization of Begmouinusamplification from leaf samples using universal primers PAL1v1978/ PAR1c715 on $1 \%$ agarose gel. M, $1 \mathrm{~kb}$ marker DNA (Thermo Scientific, US); K +, DNA of PepYLCIV ; 1-15 leaf samples from fields (1, Tegal 1; 2, Tegal 2; 3, Klaten 1; 4, Klaten 2; 5, Sleman 1; 6, Sleman 2; 7, Magelang 1; 8, Magelang 2; 9 , Magelang 3; 10, Subang 1;11, Subang 2;12, Subang3; 13, Subang 4; 14, Bogor 1; 15, Bogor 2)

but not of Cucumber Mosaic Virus (CMV). Mosaic vein banding symptom caused by Pdyrins infection had been described previously by $\mathrm{D}$ amayanti $\&$ al. (2009). This result indicated the association of Begmoinus with yellow mosaic disease of yard long bean in Java.

\section{Analysis of Sequence Identity of Begomovirus Infecting Yard Long Bean}

Nucleotide sequences were obtained for Begmovinusisolates from Tegal, Klaten, Magelang, Subang and Bogor. The sequence of isolate from Sleman was not good due to unreadable chromatogram, therefore it was not included in the further sequence analysis. Analysis of their identity by comparing to sequences on the $G$ eneBank showed their highest homology with Mungbean Yellow Mosaic India Virus (MY MIV) from Brebes and Purwakarta, i.e. $>92 \%$, followed by MYMIV from Bangladesh, Nepal, Pakistan and India, i.e. $>87 \%$ (Table 2). Their homology to MYMV, another virus causing yellow mosaic disease in South Asia, was only $71-76 \%$ and to other Begmoinus reported from Indonesia was even lower i.e. 51- 54\%. Further dendogram analysis to study their relationship showed that all 
Table2. Nucleotide sequence homology (\%) of Begmovinusinfecting yard long bean in Java with other Begmouinsesreported earlier in GeneBank

\begin{tabular}{lcccccccccccc}
\hline \multirow{2}{*}{$\begin{array}{l}\text { Begamoinusinfecting } \\
\text { yard long bean }\end{array}$} & \multicolumn{10}{c}{ Begomoinusisolates from G eneBank ${ }^{\mathrm{a}}$ ) } \\
\cline { 2 - 14 } & 1 & 2 & 3 & 4 & 5 & 6 & 7 & 8 & 9 & 10 & 11 & 12 \\
\hline pTgl1 & 99.7 & 97.9 & 92.5 & 91.1 & 92.2 & 92.1 & 76.0 & 55.8 & 53.8 & 51.4 & 51.5 & 16.6 \\
Tegal 2 & 95.7 & 94.7 & 89.5 & 88.3 & 89.4 & 89.3 & 73.1 & 54.6 & 53.9 & 52.5 & 52.0 & 17.4 \\
Klaten & 96.0 & 93.2 & 88.0 & 86.8 & 87.9 & 87.9 & 71.9 & 54.1 & 54.2 & 52.9 & 51.9 & 17.2 \\
Magelang & 95.9 & 92.8 & 87.7 & 86.4 & 87.5 & 87.5 & 71.7 & 53.5 & 53.8 & 52.1 & 51.6 & 16.8 \\
Magelang 2 & 95.3 & 92.8 & 87.7 & 86.4 & 87.5 & 87.5 & 71.7 & 53.5 & 53.8 & 52.1 & 51.6 & 16.8 \\
Subang & 95.5 & 94.7 & 89.5 & 88.3 & 89.4 & 89.3 & 73.0 & 54.6 & 54.0 & 52.5 & 52.0 & 17.4 \\
Bogor 1 & 95.4 & 94.8 & 89.6 & 88.4 & 89.5 & 89.4 & 73.0 & 54.7 & 54.1 & 52.5 & 52.2 & 17.4 \\
Bogor 2 & 95.1 & 93.8 & 88.6 & 87.4 & 88.5 & 88.4 & 72.6 & 53.7 & 54.1 & 52.7 & 52.1 & 17.5 \\
\hline
\end{tabular}

a) 1= MYMIV Brebes (JN368436); 2= MYMIV Purwakarta (JN368434); 3= MYMIV India (KC852204); 4= MYMIV Bangladesh (AF314145); 5= MYMIV Nepal (AY271895); 6= MYMIV Pakistan (AM992618); 7= MYMV India (KC911271); 8= PepYLCIV (AB246170); 9= TLCIV (AB241671), 10= ToLCNDV (dataunpublished); 11= ToLCJV (189848); 12= BCMV Bogor(FJ653916)

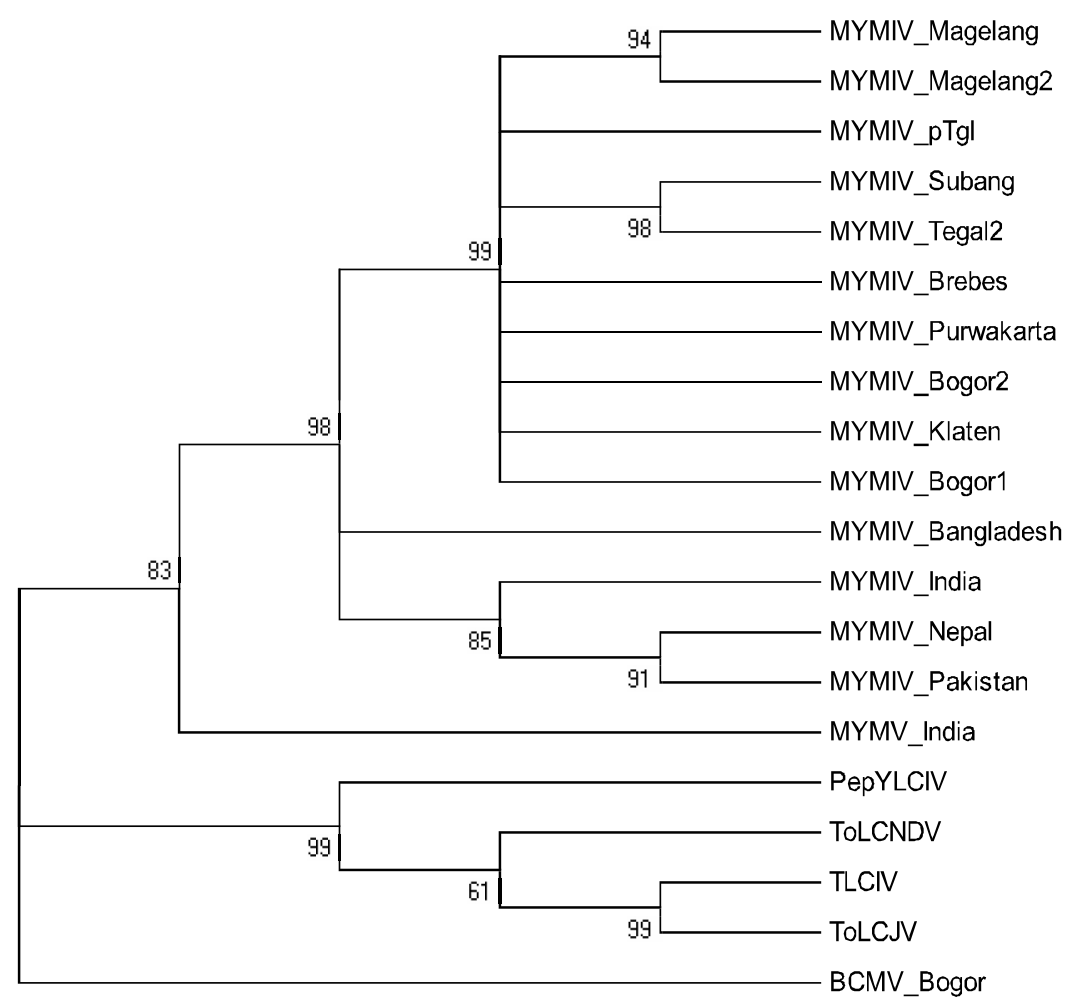

Figure 3. Phylogenetic analysis of Mungbean Yellow Mosaic India Virus based on alignment of partial nucleotide sequences of theD NA-A of Begmoinzesusing Mega 6.06 (Algorithm NeighborJoining with 1,000 bootstrapsreplicates)

Begmovinus infecting mungbean (MYMIV and MY MV) belonged to similar cluster and they were separated from Begmovinusesinfecting other crops (PepYLCIV, TLCIV, TYLCNDV, ToLCJV) (Fig. 3). It indicated that MY MIV from Java had closer genetic relationship to MYMIV from South Asia than other Begomovinuses from Indonesia.

\section{Analysis of Common Region Sequences of Begomovirus Infecting Yard Long Bean}

Molecular characters of Begmovinus could be determined by analyzing the top region of its genome covering common region and intergenic region representing the conserved and diversed region, respectively. Common region for each Beegmoinuswas different, except loop region on 


MYMIV_pTgl
MYMIV_Tegal
MYMIV_Klaten
MYMIV_Magelang1
MYMIV_Magelang2
MYMIV_Subang
MYMIV_Bogor1
MYMIV_Bogor2
MYMIV_Brebes
MYMIV_Purwakarta
MYMIV_India
MYMIV_Bangladesh
MYMIV_Nepal
MYMIV_Pakistan
MYMV_India
PepYLCIV
TLCIV
TOLCNDV
TOLCJV
BCMV_Bogor

MYMIV_pTgl MYMIV_Tegal MYMIV_Klaten MYMIV_Magelang1 MYMIV_Subang MYMIV_Bogor1 MYMIV_Bogor2 MYMIV_Brebes MYMIV_Purwak MYMIV India MYMIV_Nepal MYMIV_Pakistan MYMV_India PepYLCIV TLCIV TOLCNDV TOLCJV BCMV_Bogor

MYMIV_pTg 1 MYMIV Tegal MYMIV_Klaten MYMIV_Magelangl MYMIV Magelang2 MYMIV Subang MYMIV Bogor MYMIV_Bogor MYMIV Brebes MYMIV Purwakarta MYMIV India MYMIV_Bangladesh MYMIV_Nepal MYMIV_Pakistan MepYLCIV PepYLCI
TLCIV TOLCNDV TOLCNDV
TOLCJV BCMV_Bogor

MYMIV_pTgI MYMIV_Tegal MYMIV_Klaten MYMIV_Magelang1 MYMIV_Magelang2 MYMIV_Subang MYMIV_BOgor MYMIV_Bogor MYMIV_Brebes MYMIV India MYMIV Bangladesh MYMIV_Nepal MYMIV_Pakistan MYMV Indi PepYLCIV TLCIV TOLCND BCMV Bogor MYMIV_Magelang MYMIV Purwakarta

\begin{tabular}{|c|c|c|c|c|}
\hline 5 & & & 35 & 45 \\
\hline -GAAGTC & GTTTTTGTAT & CGGTGTA-CA & $\mathrm{CCG}$ & ТСТСТАСССС \\
\hline -GAAGTC & GTTTTTGTAT & CGGTGTA-CA & CCGATTGC-T & ТСТСТАСССС \\
\hline -GAAGTC & GTTTTTGTAT & CGGTGTA-CA & CCGATTGC-T & ТСТСТАСССС \\
\hline -GAAGTC & GTTTTTGTAT & CGGTGTA-CA & CCGATTGC-T & ТСТСТАСССС \\
\hline -GAAGTC & GTTTTTGTAT & CGGTGTA-CA & CCGATTGC-T & тСтстАСССС \\
\hline -GAAGTC & GTTTTTGTAT & CGGTGTA-CA & CCGATTGC-T & ТСТСТАСССC \\
\hline -GAAGTC & GTTTTTGTAT & CGGTGTA-CA & CCGATTGC-T & ТСТСТАСССС \\
\hline -GAAGTC & GTTTTTGTAT & CGGTGTA-CA & CCGATTGC-T & ТСТСТАСССС \\
\hline -GAAGTC & GTTTTTGTAT & CGGTGTA-CA & CCGATTGC-T & ТСТСТАСССС \\
\hline -GAAGTC & GTTTTTGTAT & CGGTGTA-CA & CCGATTGC-T & ТСТСТАСССС \\
\hline - AAAGTC & GTTTTGGTAT & CGGTGTA-CA & CCGATTGCCT & TСTCTAGCCC \\
\hline -GCAGTC & GTTTTTGC-- & $---T G T A-T A$ & CCGATTGC-T & ТСТСТАСССС \\
\hline -GAAGTC & GTTTTTGTAT & CGGTGTA-CA & CCGATTAC-T & ТСТСТАТССС \\
\hline AAGTC & GTTTTTGTAT & CGGTGTA-CA & CCGATTAC-T & ТСТСТАТССС \\
\hline TGGCGTC & GTTTT-GTAT & CGGTGTCTCT & CTAAAAGTCC & TATGTATCGG \\
\hline AGTGTC & GTTTTGTATT & GGAGACAATC & АСтTСТATCD & СTATGTATTG \\
\hline -CTTGG- & -- TCA-АTC & GGGTCTCAAC & AAACTTGCGT & TA-GCAATCG \\
\hline - CTTGTC & GTTTTG-ATT & TGACGTCCCT & СААСТTССTС & TATGTAATTG \\
\hline 80 & $--T C$ & GGAGACATTC & ATA & TG-TCAATTG \\
\hline GGGTA & АAССАСАСТT & TGTTGTTGCC & ACCAATATCA & TCGAGAACGG \\
\hline
\end{tabular}

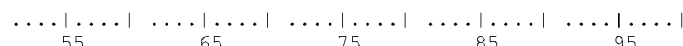
CCtATCGgT - ----GTATCG gtgtactata tatactagag ctattaAaAa CCTATCGGT- ----GTATCG GTGTACTATA TATACTAGAG CTATTAAAAG CCTATCGG TATACTAGAG CTATTAAAAG CCIATCGGT- ----GTATCG GTGTACTATA TATACTAGAG CTATTAAAAG CCIATCGG - TIATCG GIGTACTATA TATACTAGAG CTATTAAAAG CCIACCGI CCIATCGGI- ----GIATCG GIGTACTAIA TAIACTAGAG CIAITAAAAG CCIATCGGI- ---GIAICG GIGTACTATA TAIACTAGAG CTATTAAAAG CCTATCGGT - ----GTATCG GIGTACIAIA TATACTAGAG CTATIAAAAG CCTATCGGT - ----GTATCG GTGTACTATA TATACTAGAG CTACTAAAAG CCTATCGGT- ---ATATCG GTATCTTGTATATACTAGAG CTACTAAAAG CCTATCGGT- ----GTATCG GTGTCTTATA TATACTAGAA CTACTAAAAG CCTATCGGT- ---GTATCG GTGTCTTATA TATACTAGAG CTACTAAAAG TGTATCGGT - ----GTCTTA TT----TATA TGTAGGAGAG TTACTAAAAGAGA-CAGGA GACAATATAT A--TA.GTCC TATAATGGCT TTTAAGTAAT GGGAATGGGT CCCAATATAT A-GTGAGGAC CTAAATGGCA TTATTGTAAT GCGTCTGGCG TCCCATATAT ATGTAGGACG CTAAACGGCA GGATTGTAAT GAGA-CAGGA GACAATATAT A-G-GTGTCT CCAAATGGCA TAGTCGTAAT

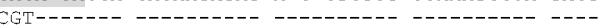

$\ldots|\ldots| \ldots|\ldots| \ldots|\ldots| \ldots|\ldots| \ldots|\ldots| \ldots \mid \ldots$ $\begin{array}{ccccc}105 & 115 & 125 & 135 & 145 \\ \text { CCCATAGGGG } & \text { CACTCAG-CT } & \text { ATAA-TATTA } & \text { CCTGAGTGCC } & \text { CCGCGACCGG }\end{array}$ CCCATAGGGG CACTCAG-CT ATAA-TATTA CCTGAGTGCC CCGCGACCGG CCCATA GGGG CACTCAG-CT ATAA-TATTA CCTGAGTGCC CCGCGACCGG CCCATAGGGG CACTCAG-CT ATAA-TATTA CCTGAGTGCC CCGCGACCGG CCCATAGGGG CACTCAG-CT ATAA-TATTA CCTGAGTGCC CCGCGACCGG CCCATAGGGG CACTCAG-CT ATAA-TATTA CCTGAGTGCC CCGCGACCGG CCCATAGGGG CACTCAG-CT ATAA-TATTA CCTGAGTGCC CCGCGACCGG CCCATAGGGG CACTCAG-CT ATAA-TATTA CCTGAGTGCC CCGCGACCGG CCCATAGGGG CACTCAG-CT ATAA-TATTA CCTGAGTGCC CCGCGACCGG CCCATAGGGG CACTCAG-CT ATAA-TATTA CCTGAGTGCC CCGCGACCGG CCTCTAGGGG CACTCAG-CT ATAA-TATTA CCTGAGTGCC CCGCGACCGG CCCATAGGGG CACTCAG-CT ATAA-TATTA CCTGAGTGCC CCGCGACCGG TCCATAGGGG CACTCAG-CT ATAA-TATTA CCTGAGTGCC CCGCGACCGG CCCATAGGGG CACTCAG-CT ATAA-TATTA CCTGAGTGCC CCGCGACCGG CCCTCA GGGG TCCTCAG-CA ATAA-TATTA CCTGAGGACC CCGCGACCGG CCCTCAGGG TCCTCAG-CA ATAA-TATTA CCTGAGGACC CCGCGACCGG ITGTACACC A-COAG

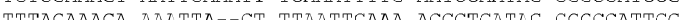

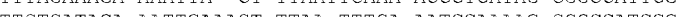
TTCTCATAGA AATTCAAACT TTAA-TTTGA AATCCAAAAG CGGCCATCCG

\begin{tabular}{|c|c|c|c|c|}
\hline 155 & 165 & 175 & 185 & 195 \\
\hline ATTGGGG & GCTTTAA- & TTAATGCT & TTTTTGGTAC & ССТTATCTTT \\
\hline TTGGGG & GCTTTAA- & DTAATGCT & TTTTTGGTAC & CCTTATCTTT \\
\hline TATTGGGG & TTGCTTTAA- & CTTTAATGCT & TTTTTGGTAC & ССTTATCTTT \\
\hline TATTGGGG & TTGCTTTAA- & CTTTAATGCT & TTTTTGGTAC & CСTTATCTTT \\
\hline TATTGGGG & TTGCTTTAA- & CTTTARTGCT & TTTTTGGTAC & ССTTATCTTT \\
\hline TATTGGGG & ПTGCTTTAA- & CTTTAATGCT & TTTTTGGTAC & CCTTATCTTT \\
\hline TATTGGGG & TTGCTTTAA- & CTTTAATGCT & TTTTTGGTAC & ССТTATCTTT \\
\hline TATTGGGG & TTGCTTTAA- & CTTTAATGCT & TTTTTGGTAC & CСТTATCTTT \\
\hline TATTGGGG & TTGCTTTAA- & CTTTAATGCT & TTTTTGGTAC & ССТTATCTTT \\
\hline TATTGGGG & TTGCTTTAA- & CTTTAATGCT & TTTTTGGTAC & CСTTATCTTT \\
\hline TATTGGGG & TTGCTTTAG- & CTTTTCTGCT & TTTTGGGTAC & ССTTATCTTT \\
\hline GTATTGGGG & TAACTTTAA- & CTTTTGTGCT & TTTTTGGTAC & ССТTATCTTT \\
\hline TATTGGGG & TTACTTTAA- & CTTTTGTGCT & TTTGTGGTAC & ССTTATCTTT \\
\hline GTATTGGGG & TTACTTTAA- & CTTTTCTGCT & TTTGTGGTAC & ССTTATCTTT \\
\hline TAC- & -GCTTTGC- & TTTT---GCT & TTTGGACCCA & ССТTATCTTT \\
\hline ГААТАТТА & CCGAGTGCCG & CGAAAATATT & TAAATGTGGT & CCCCCAAGCC \\
\hline AATATTA & CCGGATGGC- & CGCGATTTTT & TTAAAGTGGT & CCCCCCCACT \\
\hline АТАТTA & ATGAC- & CGCGCAAATT & TTTATGTGGG & СсCTC \\
\hline 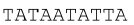 & CGGATGGC- & CGCGATTTTT & TTAAAGTGGT & ССССТССACT \\
\hline
\end{tabular}

Figure 4. Alignment of nucleotide sequences of the CR of Mungbean Yellow Mosaic India Virus (MVMIV) isolates from Java with other reported MYMIV in G enebank. The alignment showing repetitive sequences (grey shadow), invert repeat (green shadow), TATA sequences (bold letter with purple shadow) and the hairpin loop region (underlined letter with yellow shadow) with nonanucleotide sequence (bold and underlined letter with turquoise shadow) 


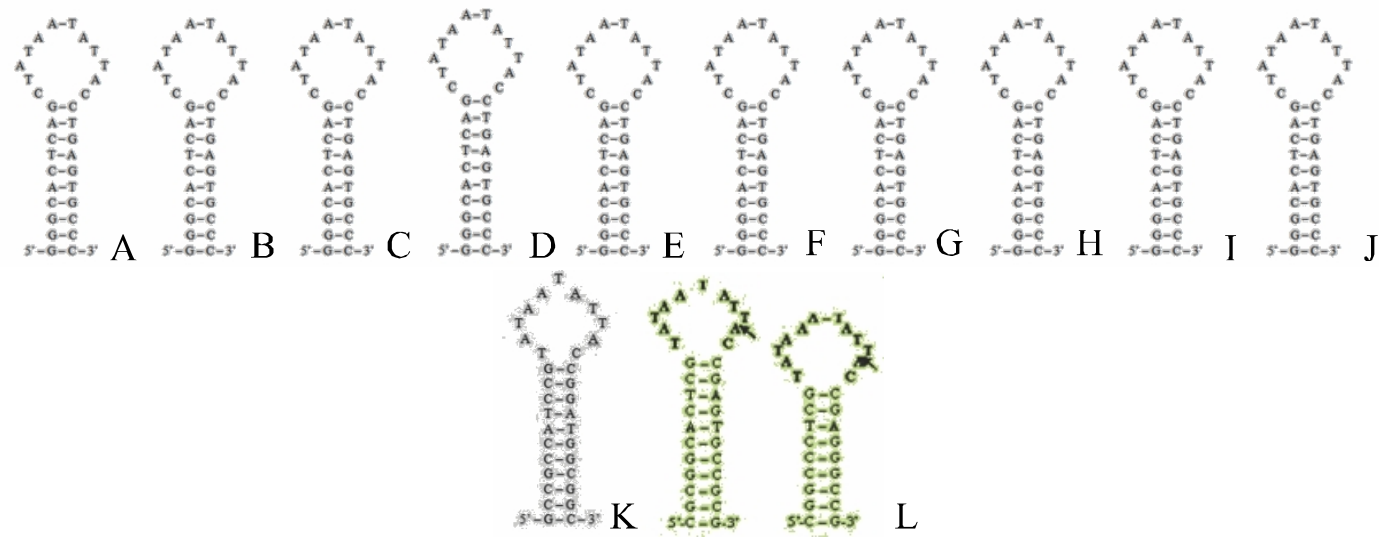

Figure 5. Hairpin loop structures of several Begomoinus isolates. A. MYMIV Tegal; B. MYMIV Klaten; C. MY MIV Magelang; D. MY MIV Subang; E. MYMIV Purwakarta; F. MYMIV Bogor; G. MY MIV Bangladesh; H. MY MIV Pakistan; I. MY MIV India;J. MY MIV Nepal; K. TLCIV Indonesia; L. PepY LCIV Indonesia

hairpin structure, 5'-TAATATTAC-3' which is known as the conserved nonanucleotide region (Lazarowitz 1987).

Comparison of iteron and protein products variations have been used to determine of MYMV and MYMIV from several regionals in India (Varma \& Malathi 2003; Usharani \&al. 2004) and TYLCV isolates from Israel, Sardinia and Thailand (Argüelo-Astorga $\&$ al. 1994). However, exception was reported on several cases, for example Potato Yellow Mosaic Virus with TYLCV-Sardinia and BGMV-Brazil with TYLCV-Israel have identical iterons although they have a rather far distance relationship (Argüelo-Astorga \&al. 1994).

O ur analysis showed that common region of Begomoinusisolates infecting yard long bean in Java consists of repetitive sequences (ATCGGTGT), TATA box, and hair pin loop structure (Fig. 4). Three direct repeats (two of them are tandem repeat sequences) can be found before TATA box in all MY MIV and MYMV isolates (Fig. 4). Iteron and TATA box sequence always present together in CR of each Begomovirus species (Lazarowitz 1987). The function of iteron and TATA box was described by Argüelo-Astorga eal. (1994) as initiator for rolling circle replication which was known as RAP-specific binding sites. Similar characteristics were reported for Eastern Hemisphere geminivirus except ACMV and ICMV, Western Hemisphere geminivirus, and SqLCV-E and - R (Argüelo-A storga $\&$ al. 1994), and MYMIV on soybean (Usharani \& al. 2004).
Hair pin loop structure of each isolates was identical to MY MIV from Bangladesh, Pakistan, India, Nepal, and one isolate of PepY LCIV, but they were different from TLCIV and PepY LCIV (Fig. 5). Begmovinus possessing $>92 \%$ similarity on their hairpin loop structure could be grouped as one species (Fauquet $\notin$ al. 2005; Hidayat $\notin a$ al. 2008). Therefore, all Begmovinusisolates from this study was the same species with MY MIV from Bangladesh, India, Pakistan, and Nepal.

\section{CONCLUSIONS}

Molecular detection and characterization confirmed the association of MYMIV in yellow mosaic disease of yard long bean in Java. Virus isolates from Java had the highest similarity (>85\%) with MYMIV isolates from Bangladesh, Pakistan, India, and Nepal. Further study on characters of the virus, such as host range, insect transmission and disease spread, are very important to understand disease development and control.

\section{ACKNOWLEDGEMENTS}

This research was funded by collaboration between AUSAID funded Economic Cooperation Work Program of the ASEANAustralia-New Zealand Free Trade Agreement and D epartment of Plant Protection, Faculty of Agriculture, InstitutPertanian Bogor. 


\section{REFERENCES}

Aidawati N, Hidayat SH, Suseno R, Hidayat P, Sujiprihati S. 2005. Identifikasi gminiunusyang menginfeksi tomat berdasarkan pada teknik polymease dain readionrestridion fragnent lengh pdymorphism J Mikrobiol Indon 10(1): 29-32.

Argüelo-Astorga GR, Guevara-González RG, HerreraEstrella LR, RiveraBustamante RF. 1994. Geminivirus replication origins have a groupspecific organization of iterative elements: a model for replication. Virology 203:90100.

D amayanti TA, Alabi OJ, Rauf A, Naidu RA. 2009. Severe outbreak of yellow mosaic disease on the yard long bean in Bogor, West Java. HAYATI J of Biosci 16(2):78-82.

Doyle JJ, Doyle JJ. 1987. A rapid DNA isolation of procedure for small quantities of fresh leaf tissue. Phytochem Bull 19:11-9.

Fauquet CM, Mayo MA, Maniloff J, Desselberger U, Ball LA, editor. 2005. Vinus Taxonomy. Eight Report of the International Committee on Taxonomy of Viruses. San Diego (US): VirolDivInt Union of Microb Soc.

Hidayat SH, Chatchawankanpanich O, Rusli E, Aidawati N. 2006. Beegmoinsassociated with pepper yellow leaf curl disease in West Java, Indonesia. J Mikrobiol Indon 11:87-90.

Hidayat SH, Chatchawankapanich O, Aidawati N. 2008. Molecular identification and sequence analysis of Tdbacco Lef Cud Vins from Jember, East Java, Indonesia. HAYATIJ of Biosci 15(1):13-7.

Hull R. 2002. Matthew's PlantVirdogy $4^{\text {th }}$ Ed London (GB): Academic Press.

Ilyas M, Q azi J, Mansoor S, Briddon RW. 2010. G enetic diversity and phylogeography of Besomoinuses infecting legumes in Pakistan. J G en Virol [Internet]. [cited 2012 Apr 24]; 91:2091-2101. Available from: http:/ / www.pubfacts.com/ detail/ 20375225/ G ene tic-div ersity-and - phylo geo grap hy- of begomoviruses-infecting-legumes-in-Pakistan. D O I: 10.1099/ vir.002 0404-0.

Lazarowitz SG.1987. The molecular characterization of geminivinuses Plant Mol Biol Rep 4:177-92.

National Centre for Biotechnology Information. 2013. [cited 2013 Jun 20]; Available from http:/ / www.ncbi.nlm.nih.gov.

Rojas MR, Gilbertson RL, Russel DR, Maxwell D P. 1993. Use of degenerate primers in the polymerase chain reaction to detect whitefly-transmitted geminivinuses Plant Dis [Internet]. [cited 2012 Apr 24]; 77(4): 340-47. Available from: http:// www. apsnet.org/ publications/ plantdisease/ backissues/ D ocuments/1993Articles/ PlantD isease77n04_340 .pdf.

Shahid MS, Pudashini BJ, Khatri-Chhetri GB, Ikegami M, Natsuaki KT. 2012. First report of kidney bean in Nepal. New Dis Rep [Internet]. [cited 20130 ct 22]; 25:30. D OI:10.5197/j.2044-0588.2012.025.030. Available on: http:// www.ndrs.org.uk/ article.php ?id $=025030$.

Tsai WS, Shih SL, Rauf A, Safitri R, Hidayati N, Huyen BTT, Kenyon L. 2013. G enetic diversity of legume yellow mosaic Besmoinuses in Indonesia and Vietnam. Ann Appl Biol 163:367-77.

Usharani KS, Surendranath B, Haq QMR, Malathi VG. 2004. Yellow mosaic virus infecting soybean in northern India is distinct from the species infecting soybean in southern and western India. Current Sci. [Internet]. [cited 2012 May 8]; 86(6):845-850. Available on: http:// www.iisc.ernet.in/ currsci/ mar252004/ 845.pdf.

Varma A, Malatthi VG. 2003. Emerging geminivirus problems: a serious threat to crop production. Ann Appl Biol. [Internet]. [cited 2014 Apr 3]; 142:145164. Available on: http:/ / onlinelibrary.wiley.com/ doi/ 10.1111/ j.17447348.2003.tb00240.x/ pdf. 